\title{
The Potential of Single Shot Dynamic TEM for Combined Nanosecond and Nanoscale In-Situ Microscopy
}

\author{
N. D. Browning ${ }^{* * *}$, M. R. Armstrong ${ }^{*}$, G.H. Campbell ${ }^{*}$, J. S. Kim ${ }^{*, * *}$, W. E. King ${ }^{*}$, T. B. LaGrange , \\ C. J. Mitterbauer**, and B. W. Reed \\ * Materials Science and Technology Division, Lawrence Livermore National Laboratory, \\ Livermore, CA 94550, USA \\ ** Department of Chemical Engineering and Materials Science, University of California, Davis, CA \\ 95616
}

\begin{abstract}
Aberration correctors for higher spatial resolution TEM/STEM and monochromators for higher spatial resolution TEM and higher energy resolution electron energy loss spectroscopy (EELS) have dominated much of the instrument development that has taken place in electron microscopy over the last few years. However, while these new components have revolutionized the ability to record static information from the TEM/STEM, they have done little to improve our ability to measure the dynamics of materials processes. To address the need to understand the interactions that take place in materials during synthesis, deformation and under transient applied conditions, work performed at Lawrence Livermore National Laboratory (LLNL) is developing a dynamic TEM (DTEM) with the ability to record images with nanosecond time resolution and nanoscale spatial resolution [1].
\end{abstract}

This work has built on the pioneering developments of Bostanjoglo and co-workers [2] in using a photoemission source to create a short pulse of electrons in the microscope column. Experiments are performed by using one laser to "pump" the specimen and another laser to produce the pulse of electrons at the source that then travels down the column to "probe" the response of the sample (Figure 1a). By varying the time interval between the pump and the probe, the dynamics of the processes created by the pump laser can be investigated. The probe electrons are used to obtain either a diffraction pattern, or if there are sufficient electrons in the pulse, to form an image. One of the key elements of the DTEM project is the ability to generate and manipulate electron pulses with enough electrons to form single-shot images. It is the ability to perform single-shot experiments that differentiates this approach from other attempts to perform dynamic experiments in the TEM [3]. As the majority of processes in solid materials take place at specific locations and are not uniformly reproducible over many cycles, it is this ability to obtain an image through a single exposure that offers the most potential applications. As such, the DTEM at LLNL is currently being implemented to analyze phase transitions, dislocation dynamics, reactive multilayers, and nanoscale particles.

There are many issues that affect the ultimate resolution of the DTEM, such as signal-to-noise limitations, spatial and temporal incoherence, lens aberrations, and electron-electron interactions (space charge broadening and stochastic trajectory displacements). Many of these effects are exacerbated by the design of current microscope columns, which are optimized in performance for a single electron in the column at any given time and not the $\sim 10^{6}$ electrons in a nanosecond pulse that exist in the DTEM. However, despite these limitations, the DTEM based on the JEOL 2000FX at LLNL can already produce images with $<20 \mathrm{~nm}$ spatial resolution and $\sim 1.5 \mathrm{~ns}$ temporal resolution (Figure 1b). Given that there are several straightforward ways to modify the electron column, such resolution at this early stage of development holds much promise for the future of these methods. 
It should be noted that the temporal resolution of the instrument is primarily governed by the pulse width of the laser used to excite the source (although broadening does occur when the pulse duration is very short or there are a lot of electrons in the pulse). This means that any time resolution below the current limit of $\sim 1.5 \mathrm{~ns}$ can currently be achieved in single shot images on the DTEM. Using an in-situ stage (Figure 2) for the DTEM (designed by Fischione) this means that traditional in-situ experiments can routinely be performed in the $\mu$ s to ns time regime. In this presentation, the current limitations and future expectations for in-situ microscopy in the DTEM will be discussed and the first results from the LLNL instrument will be presented [4].

[1] W. E. King et al, J. Appl. Phys. 97 (2005) 111101

[2] H. Dömer and O. Bostanjoglo, Rev. Sci. Inst. 74, 4369-4372 (2003)

[3] V. A. Lobastov et al, Proc. Nat. Acad. Sci. USA. 102, 7069 (2005)

[4] Work performed under the auspices of the U.S. Dept. Energy, Office of Basic Energy Sciences by University of California, Lawrence Livermore National Laboratory under Contract W-7405Eng-48.

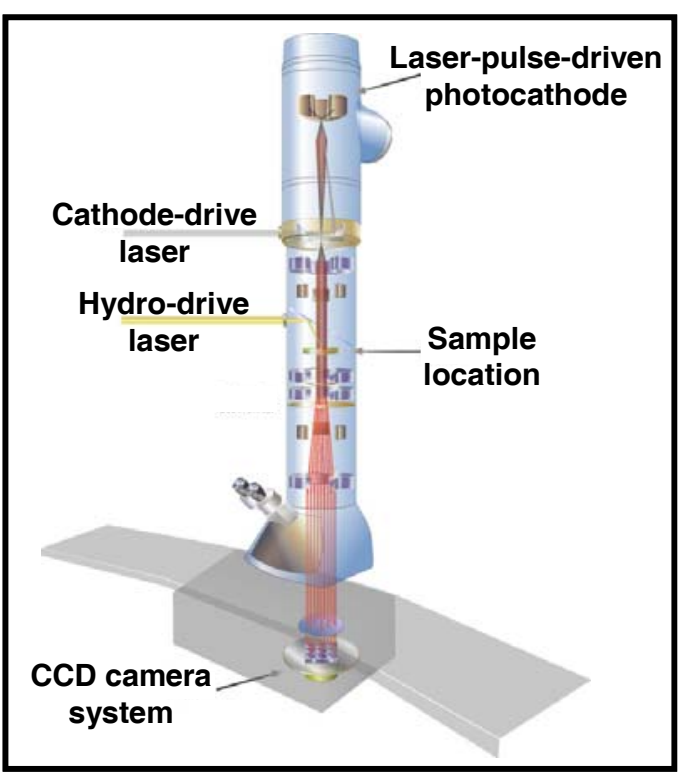

Figure 1: (a) Schematic of the DTEM at LLNL (b) Diffraction grating replica image demonstrates a spatial resolution of below $20 \mathrm{~nm}$.
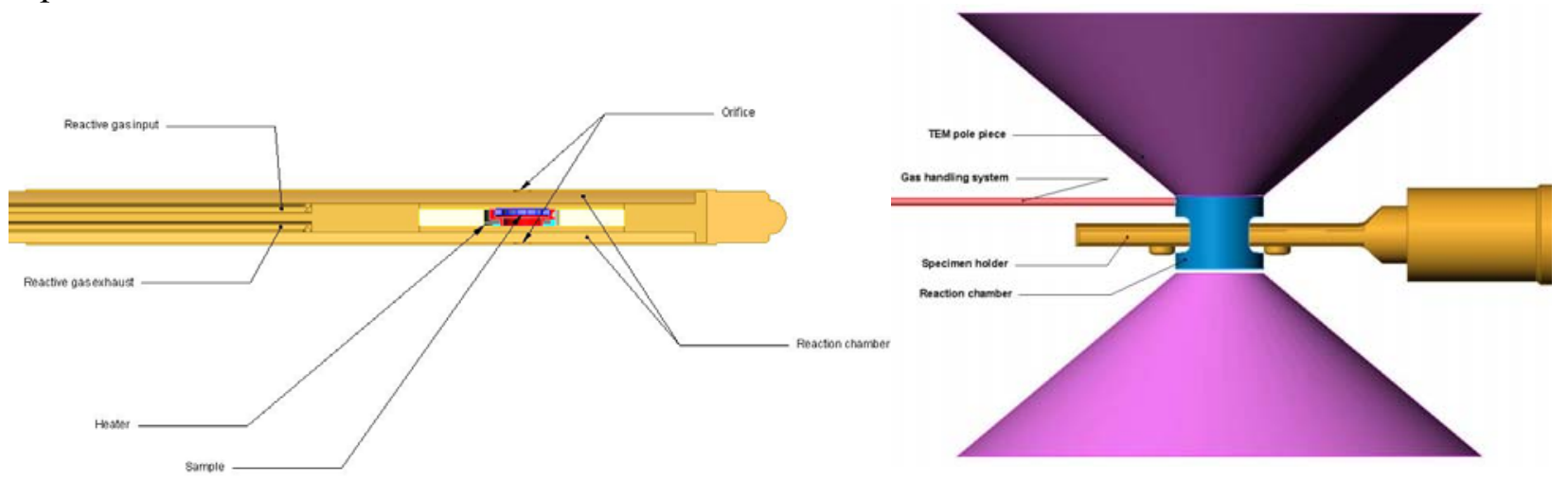

Figure 2: (a) The specimen holder will permit annealing under a variety of gas conditions prior to insertion where (b) a gas handling stage will allow the environment to be precisely controlled. 